\title{
STABILITY ANALYSIS OF A VISION-BASED CONTROL DESIGN FOR AN AUTONOMOUS MOBILE ROBOT ${ }^{1}$
}

\author{
J.B. Coulaud, M. De Wan, G. Bastin, G. Campion \\ CESAME, UCL, B1348 Louvain-La-Neuve, Belgium, \\ coulaud@auto.ucl.ac.be,dewan@auto.ucl.ac.be, \\ bastin@auto.ucl.ac.be,campion@auto.ucl.ac.be
}

\begin{abstract}
We propose a simple control law allowing a mobile robot equipped with a low cost camera to track a line drawn on the ground. The control algorithm as well as the image processing algorithm are very simple. We discuss the existence and the stability of an equilibrium configuration of the robot when tracking a circular reference line. We then give a short extrapolation to more general reference curves. Experimental results confirm the theoretical analysis. Copyright (C)2005 IFAC
\end{abstract}

\section{INTRODUCTION}

The problem we address is the feedback control design allowing a mobile robot to track a line drawn on the ground. The position of the line with respect to the robot is detected by an embarked video camera. There are a lot of image processing algorithms extracting a map of the environment from the data provided by a camera (see for instance (A. Broggi, 1999), (Dickmanns, 1994), (C. Thorpe, 1991), (D. Pomerleau, 1996) and (C. J. Taylor, 1999)). But the implementation of such sophisticated algorithms is quite complex. Moreover, there exist several approaches to design a control allowing to track a reference trajectory. For instance, the differential flatness of the robot allows to reduce the problem to a path planning problem (see (A. Piazzi, 2002), (M. Fliess, 1999), (P. Rouchon, 1993)). But these approaches require a highly accurate on-line extraction of the line shape. An other efficient method is given by (L.P. Lapierre, 2003), whose advantage is to have a control design that avoids singularities, but the

\footnotetext{
1 This paper presents research results of the Belgian Programme on Interuniversity Attraction Poles, initiated by the Belgian Federal Science Policy Office. The scientific responsibility rests with its authors.
}

restriction is that this approach needs the vision sensor to be able to see the path everywhere around and overall under the robot. This is a quite strong assumption that is hardly ever verified by vision based mobile robots.

Our purpose in this communication is to propose a simple solution of this tracking problem which avoids as much as possible sophisticated image processing and control algorithms. The image processing is reduced to the identification of a single target point on the ground, and the control design is quite natural: a constant forward velocity is imposed while the angular velocity is proportional to the distance of the target point from the longitudinal axis of the robot. The practical implementation is straightforward and can easily be achieved on line. Our main contribution is to provide a complete stability analysis of the control system. Restricting ourselves, in a first step, to a particular reference trajectory (a circle) we show that there is an asymptotically stable equilibrium configuration for the robot with respect to the line, and we explicit the domain of attraction of this equilibrium. Then, in a second step, this result is extended to the analysis of the performances of the closed loop system for arbitrary line shapes. 


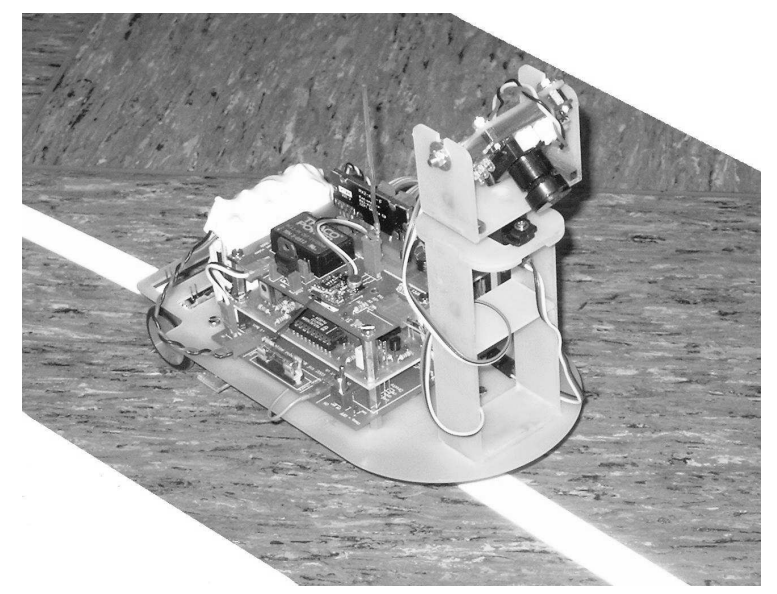

Fig. 1. The experimental robot

We first describe the experimental device and the robot kinematic model (Sections 2 and 3). We then define the control law (Section 4). Section 5 is devoted to the existence and stability of an equilibrium configuration around a circular line, and the behavior of the system for arbitrary reference line with upper bounded curvature. We end this part of the study with a possible improvement (Section 6). Section 7 gives a short explanation of the image processing and Section 8 shows an overview of experimental results.

\section{EXPERIMENTAL DEVICE}

The mobile robot is of unicycle type: it is equipped with two fixed wheels at the back and a caster wheel at the front, which has no influence on the kinematic properties. The length of the robot is $22 \mathrm{~cm}$ and the distance between the fixed wheels is $7.4 \mathrm{~cm}$. A speed control is provided for the two fixed wheels, from a computer using Labview which transmits the latches.

The vision device is constituted by a low cost monochromatic camera with a resolution of $320 \times$ 240. It is fixed on the robot, at a height of $17 \mathrm{~cm}$, at a distance of $14.2 \mathrm{~cm}$ from the rear axle. It leans forward, with an angle of $45^{\circ}$. The lateral vision angle is $60^{\circ}$, which limits significantly the vision field. Data are transmitted to the computer serial port by usual TV norms.

\section{KINEMATIC MODEL}

We suppose - as it is usually done - that the contact between the wheel and the ground satisfies both conditions of pure rolling and non-slipping during the motion; moreover the robot is assumed to be rigid.
With these assumptions we can derive the expression of the well known kinematic model:

$$
\begin{aligned}
& \dot{x}=V \cos \theta \\
& \dot{y}=V \sin \theta \\
& \dot{\theta}=\omega
\end{aligned}
$$

where $(x, y)$ denote the coordinates of the middle point $P$ of the back wheels axle, $\theta$ is the angle that fixes the robot orientation in a specified Galilean base frame, $V$ is the algebraic velocity of $P$ (also called forward velocity) and $\omega$ is the angular velocity. $V$ and $\omega$ can be assigned by the physical inputs of the experimental device, i.e. the rotation speeds of the two fixed wheels.

\section{LINE TRACKING CONTROL DESIGN}

The strategy we use is quite natural: essentially it consists in controlling the orientation so that the path to track would be centered in the vision field.

On one hand, we impose the forward velocity to be constant: $V=V_{0}$. On the other hand we define a fixed horizontal line of pixels on pictures from the camera, which corresponds approximatively to a real line of the ground which is parallel to the rear axle. We will called it the horizon. The distance from the rear axle to the horizon is noted $H$. Then we consider the intersection of the horizon with the line to track. If the curvature of the line is not too high, this defines a unique point $P_{r}$, and even if there are several possible choices, we suppose that $P_{r}$ is the nearest point to the center of the field of vision.

We then evaluate the distance from $P_{r}$ to the main axis of the robot $\left(O, \overrightarrow{x_{r}}\right)$, denoted by $Z$. This situation is summed up on Fig.2. Finally we impose angular velocity to be proportional to this distance: $\omega=k Z$, where $k$ is a constant coefficient.

The closed loop system is then described by the following equations:

$$
\begin{aligned}
& \dot{x}=V_{0} \cos \theta \\
& \dot{y}=V_{0} \sin \theta \\
& \dot{\theta}=k Z
\end{aligned}
$$

\section{STABILITY ANALYSIS}

To study the stability of this control we first examine the case where the line to track is a circle. Afterwards we will extrapolate some results for any curve. 


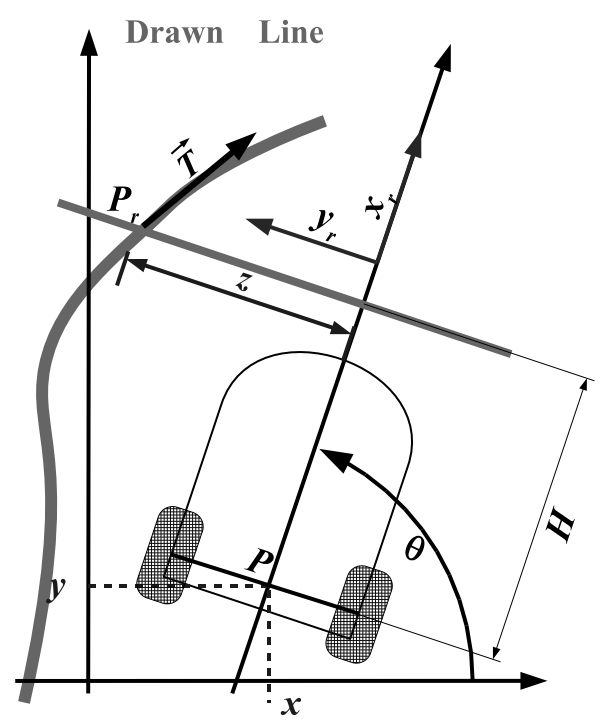

Fig. 2. Control Principle

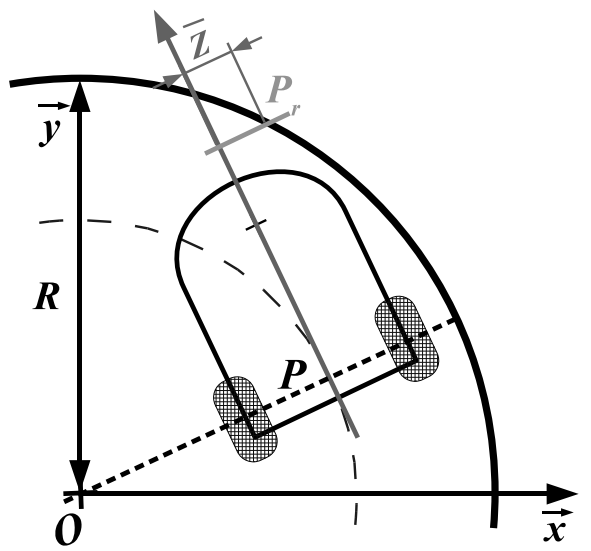

Fig. 3. Circle tracking

\subsection{Equilibrium trajectory for a circular line}

The circle to track is centered at $O$ the origin of the Galilean reference frame, with radius $R$, as shown on Fig.3.

We first study if there exists an equilibrium configuration for the robot with respect to the circle, and if this configuration is locally stable and attractive. It can be expected that, at the equilibrium, the middle point $P$ of the rear axle describes a circle, as it will be confirmed after.

From this point of view, the state variables $(x, y, \theta)$ are not the most relevant, and it is preferable to choose outputs that will converge to constant values at the equilibrium. If the robot describes a circle centered at $O$, the coordinates of $O$ in a reference frame attached to the robot are constant. So this leads to choose the two orthogonal projections $h$ and $r$ of $O$ on the axis $\left(O, \overrightarrow{x_{r}}\right)$ and $\left(O, \overrightarrow{y_{r}}\right)$ of the robot as outputs of the system. To work with non dimensional variables and simplify the equations we finally define:

$$
\begin{aligned}
u & =\frac{h+H}{R} \\
v & =\frac{r}{R}
\end{aligned}
$$

The geometrical constraint ensuring that there is an intersection between the horizon and the white line reduces to a first condition:

$$
|u| \leq 1
$$

A second condition has to be imposed in order to ensure the existence of the control law. The control is not defined if the horizon intersects the circle at two points which are equidistant from the main axis of the robot. This unacceptable situation occurs when $v=0$. This means that we do not accept sign changes for $v$. Then, without loss of generality, we consider that:

$$
v>0
$$

This simply means that the robot is moving in the anticlockwise direction around $O$.

It is easy to show that the variables $u$ and $v$ evolve according to the following equations:

$$
\begin{aligned}
& \frac{d u}{d \tau}=v \sqrt{1-u^{2}}-v^{2}+\varpi \\
& \frac{d v}{d \tau}=(u-\sigma)\left(v-\sqrt{1-u^{2}}\right)
\end{aligned}
$$

where

$$
\begin{aligned}
\sigma & =\frac{H}{R} \\
\varpi & =\frac{V_{0}}{k R^{2}} \\
\tau & =\frac{V_{0}}{\varpi R} t
\end{aligned}
$$

The only equilibrium point of this system is given by:

$$
\begin{aligned}
& \bar{u}= \\
& \bar{v}=\frac{\left.\sqrt{1-\sigma^{2}}+\sqrt{1-\sigma^{2}+4 \varpi}\right)}{2}
\end{aligned}
$$

The value of $\bar{v}$ represents the distance $O P$ at the equilibrium.

The Jacobian matrix of this system is:

$$
\left(\begin{array}{cc}
-\frac{u v}{\sqrt{1-u^{2}}} & \sqrt{1-u^{2}}-2 v \\
v-\sqrt{1-u^{2}}+\frac{u(u-\sigma)}{\sqrt{1-u^{2}}} & u-\sigma
\end{array}\right)
$$

At the equilibrium this matrix becomes:

$$
\left(\begin{array}{cc}
-\frac{1}{2}\left(\sigma+\sqrt{1+\frac{4 \varpi}{1-\sigma^{2}}}\right) & -\sqrt{1-\sigma^{2}+4 \varpi} \\
\frac{\sqrt{1-\sigma^{2}+4 \varpi}-\sqrt{1-\sigma^{2}}}{2} & 0
\end{array}\right)
$$


Its determinant is positive and its trace is negative which implies that the eigenvalues have negative real parts. So the subsystem is locally asymptotically stable around the equilibrium, for any values of $(\sigma, \varpi) \in] 0,1\left[\times \mathbb{R}_{+}^{*}\right.$

\subsection{Domain of attraction of this trajectory}

In order to characterize the domain of attraction of this equilibrium point we consider the phase plane detailed in Fig.4 where we have chosen $\sigma=0.6$ and $\varpi=0.07$. The equilibrium state is denoted by the black cross and has coordinates $(0.60,0.88)$.

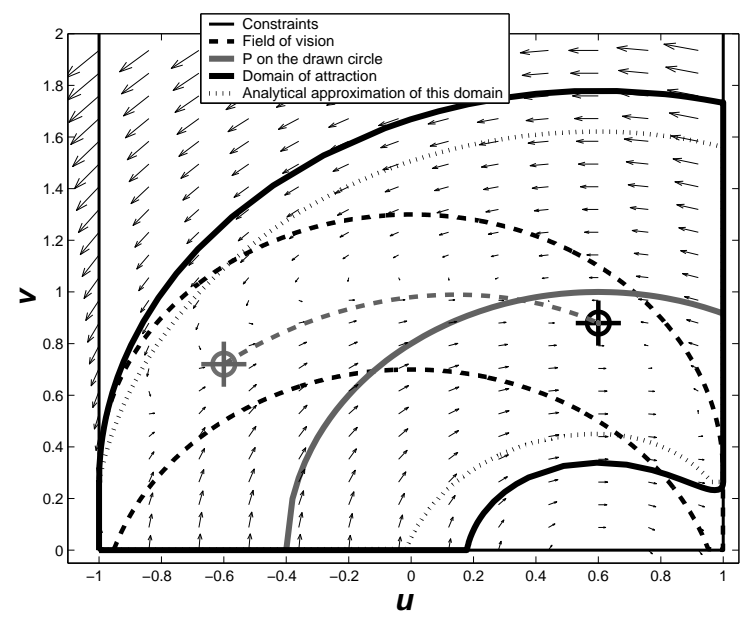

Fig. 4. Domain of attraction of the equilibrium

The domain of attraction is delimited by the black solid line: it is the largest region of the plane that satisfies the constraints (4) and (5) and for which the field of vectors always points to the interior of the delimited area. The computation is achieved by MatLaB. Nevertheless, for this system it is possible to determine analytically an approximation of the domain of attraction represented by the dotted line on the figure ${ }^{2}$.

It can be shown that the set of states $(u, v)$ corresponding to situations where $P$ is on the reference circle, is a circle arc in the plane phase, with radius 1 and centered on $(\sigma, 0)$. It is the gray solid curve on the figure.

So, on this example, parameters are such that $P$ is located inside the circle to track at the equilibrium configuration.

There is a last aspect we have to consider: the restriction of the field of vision. Considering the maximal angle of vision we define a maximum value $Z_{\max }$ for the deviation $Z$ involved in the feedback of equation (2). Considering $z=\frac{Z}{R}$ and

\footnotetext{
2 The expression is presented in annex of the following French pdf document http://www.auto.ucl.ac.be/ coulaud/memoire.pdf
}

$z_{\max }=\frac{Z_{\max }}{R}$ the expression of this variable in function of $u$ and $v$ becomes:

$$
z=v-\sqrt{1-u^{2}}
$$

The region where $|z| \leq z_{\max }$ is delimited by the black black dashed curve on the figure.

So, to make sure that the robot will converge to the equilibrium trajectory, we must impose an initial state that will produce a trajectory confined in the intersection of the black solid and black dashed areas. The set of initial points satisfying this condition is the largest stable area included in the field of vision. In practice, if

$$
\varpi \leq \frac{\sigma^{2}}{4}
$$

this region of the phase plane is very close to the intersection of the domain of attraction with the vision field one.

A good stability margin estimator is the distance from the equilibrium to the vision field boundary, whose expression is

$$
m_{s}=\frac{1-\sqrt{\sigma^{2}+\left(\bar{v}-z_{\max }\right)^{2}}}{\sigma}
$$

The division by $\sigma$ aims at keeping this margin in the same range independently of $R$. If inequality (12) is satisfied then $m_{s}$ essentially depends on the value of $\sigma$ which must be small enough. Moreover we then have $\bar{v} \leq 1$. So, having a good stability margin leads to $\sigma \ll \sqrt{1-\left(1-z_{\max }\right)^{2}}$ which can be strengthen in:

$$
H \ll \sqrt{2 Z_{\max } R}
$$

In this inequality « only means that the smallest $H$ is, the best the margin is. In any case, we recommend to choose at least $\sigma<0.7$ to keep equilibrium state far enough from the right boundary of plane phase.

In the next two paragraphs we will denote $\mathcal{D}$ the set of $(\sigma, \varpi)$ with $\sigma \in[0,0.7]$ and $\varpi=\frac{\sigma^{2}}{4}$.

\subsection{Convergence rate}

In equations (7) we introduced a characteristic time $t_{c}=\frac{\varpi R}{V_{0}}$; this is the unit time of equations (6). So during this time we can give a characteristic distance $d_{c}$ scanned by $P$, whose velocity is $V_{0}: d_{c}=V_{0} t_{c}=\varpi R$ Then, considering that $P$ is moving around the center $O$, at an approximative distance $R$ from it, we find a characteristic angle of rotation $\phi_{c}{ }^{3}$ around $O$ during $t_{c}$ :

$$
\phi_{c}=\frac{d_{c}}{R}=\varpi
$$

\footnotetext{
3 in radians
} 
This remarkable result is not sufficient to conclude that the robot will approximately converge to the stable trajectory after an angle of $\phi_{c}$, but will allow us to conclude this study by numerical tests.

We call stabilization time at 0.1, the maximal non dimensional time $T_{s}$ for which the system has reached a state $0.1 \sigma$ distant from equilibrium, in plane phase units, for any initial state $(u(0), v(0))$, and any couple $(\sigma, \varpi) \in \mathcal{D}$. Then we have an approximate angle of stabilization at $0.1 \phi_{s}=$ $T_{s} \phi_{c}$. We then compute $\phi_{s}$ for lots of values of $(\sigma, \varpi) \in \mathcal{D}$. We can see that $\phi_{s}$ has a maximum value of $\phi_{\max } \simeq 125^{\circ}$ for the "longest" trajectory in the domain of attraction. This means that, whatever the admissible initial state, the robot is very close to the equilibrium after one third of a turn around the center $O$.

\subsection{Extrapolation to any curve}

A natural question is the following: "if the line to track is such that its curvature satisfies relation (14) at every point, will the robot follow this trajectory ?" A complete answer is quite hard to give, but it is possible to study this problem by some kinematic approximations. First, knowing the geometrical parameter $H$ we note $c_{\max }=\frac{0.7}{H}$, the maximal curvature we accept for the drawn line. This is to be coherent with $\sigma<0.7$ in the previous study. We also keep

$$
(\sigma, \varpi) \in \mathcal{D}
$$

with the value $R=\frac{1}{c_{\max }}$ It is then intuitive that the most difficult curves to track will be the ones which are the most curved and which oscillate a lot. Thus, we focus on a special curve which is a succession of two circle arcs of radius $R$, with an intermediate inflexion point as described on Fig. 5 .

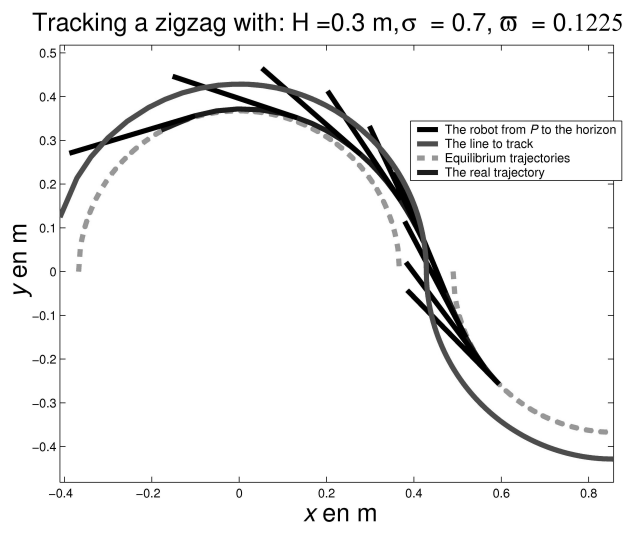

Fig. 5. A difficult line to track

We suppose that the robot is initially stabilized on the first arc equilibrium trajectory and we want to know if it will join the equilibrium trajectory of the second arc. We can look again at Fig.4 where the gray dashed trajectory represents this transition. It is important to note that if the initial state is in the vision field, the whole trajectory also satisfies this condition.

Furthermore, if we compute again a maximal stabilizing angle at $0.1 \gamma_{\max }$ for this particular trajectory, we find a value near $94^{\circ}$. We then can draw the following conclusion:

if

(1) the curvature of the line is upper bounded

(2) parameters satisfy equation (16)

(3) stability margin $m_{s}$ is acceptable

(4) two successive inflexion points $M$ and $N$ are always such that: $M N>\frac{\gamma_{\max }}{c_{\max }}$

then the robot will be able to track this line.

\section{IMPROVEMENTS OF THIS LAW}

A simple criticism we could formulate for $(\sigma, \varpi) \in$ $\mathcal{D}$ is that the point $P$ is always stabilized in the interior of the curvature, but never on the line except if it is a straight one. In order to achieve a more accurate control it can therefore be interesting to change the values of the parameters: choosing

$$
\varpi=1-\sqrt{1-\sigma^{2}}
$$

when the robot tracks a circle, stabilizes $P$ right on the line because $\bar{v}=1$. To extend this conclusion we can compute the curvature of the line to track and adapt $\varpi$ and $\sigma$ at the same time so that condition (17) will be satisfied for this curvature. Then, denoting $s$ the curvilinear coordinate and $c$ the curvature, if $\frac{d c}{d s}$ is small enough we can consider that $P$ is stabilized on the the line. So we have cancelled constraint (16) but it does not matter since the robot is always near equilibrium. We can give the following new constraint

$$
\frac{d c}{d s} \ll \frac{1}{H^{2}}
$$

\section{A SIMPLE IMAGE PROCESSING}

To keep a quick image processing we implement a simple method to find a point of the line to track. Let's choose one ore more fixed horizontal lines of pixels. As we want to track a white line we only have to select every point appearing bright enough (using a threshold for instance), and then take the middle of the first one and the last one, which will approximatively give the middle of the white line. One could notice that this is not robust if there are other white objects in the vision field. We can 


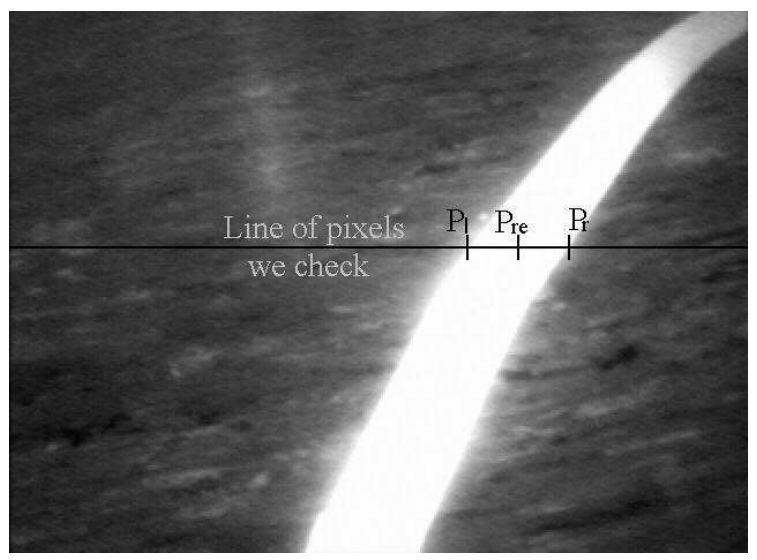

Fig. 6. Finding a point of the white line

improve this method by several simple ways like checking the width of the alleged line to verify that this is coherent with the expected width.

\subsection{Enlarging the field of vision}

The field of vision is obviously a limiting factor in our problem, because we must make sure that the white line will always be visible for the video camera. Anyway, it is important to limit the curvature of the line to track, because of mechanical constraints, but it would be preferable that the field of vision would be wide enough not to be a limiting factor.

That is why it is interesting to add a rotation on the camera to widen the angle of vision. Then, by a test on each snapshot we can impose that the camera focuses on the white line if this one is far too much from the center of the picture.

\section{EXPERIMENTAL RESULTS}

We have tested our strategy with the our robot on a white line (see Fig.7) with a minimum curvature

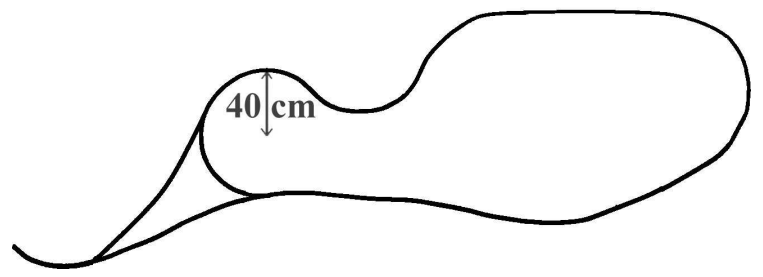

Fig. 7. The test path

radius of $40 \mathrm{~cm}$ (knowing the distance between the controlled point $P$ and the beginning of the field of vision is about $38 \mathrm{~cm}$ ) and having also some inflexion points.

We have tested the control with the following modifications:

- the camera can rotate to improve the field of vision
- the parameters are modulated so that the robot moves faster on straight lines and more slowly in the curves, with a better control of the point $P$

The distance between $P$ and the white line is always smaller than $3 \mathrm{~cm}$ and often smaller than $1 \mathrm{~cm}$. These results seem good enough compared with the intrinsic error of the approximation we made of the path; a video can be downloaded from http://www.inma.ucl.ac.be/ ${ }^{\sim}$ coulaud/

\section{CONCLUDING REMARKS}

Lots of improvements could be added to the path tracking strategy we propose. Nevertheless, the advantage of this method is that it is really simple to implement and requires few computations for a quite good accuracy of the control compared with the cheapness of the components.

\section{REFERENCES}

A. Broggi, M. Bertozzi, A. Fascioli G. Conte (1999). Automatic vehicle guidance:the experience of the argo autonomous vehicle. World Scientific.

A. Piazzi, C.G. Lo Bianco, M. Bertozzi A. Fascioli A. Broggi (2002). Quintic g2-splines for the iterative steering of vision-based autonomous vehicles. Transactions on Intelligent Transportation Systems 3, 27-36.

C. J. Taylor, J. Kos̆eckà, R. Blasi J. Malik (1999). A comparative study of vision-based lateral control strategies for autonomous highway driving. Int. J. Robot. Res. 18(5), 442-453.

C. Thorpe, M. Hebert, T. Kanade S. Shafer (1991). The new generation system for the cmu navlab. Vision-Based Vehicle Guidance pp. 83-110.

D. Pomerleau, T. Jochem (1996). Rapidly adapting machine vision for automated vehicle steering. IEEE Expert 11, 19-27.

Dickmanns, E.D (1994). The 4d-approach to dynamic machine vision. Proc. 33rd Conf. Decision and Control. pp. 3770-3775.

L.P. Lapierre, D.J. Soetanto (2003). Vision-based path following control of a unicycle robot. 11th Mediteranean Conference on Control and Automation.

M. Fliess, J. Lévine, P. Martin P. Rouchon (1999). A liebcklund approach to equivalence and flatness of nonlinear systems. IEEE Trans. Automat. Contr. 44, 922-937.

P. Rouchon, M. Fliess, J. Lévine P. Martin (1993). Flatness, motion planning and trailer systems. Proc. 32nd IEEE Conf. Decision and Control pp. 2700-2705. 